\title{
Involvement of PTEN in airway hyperresponsiveness and inflammation in bronchial asthma
}

\author{
Yong-Geun Kwak, ${ }^{1}$ Chang H. Song, ${ }^{2}$ Ho K. Yi, ${ }^{3}$ Pyoung H. Hwang, ${ }^{3}$ Jong-Suk Kim, ${ }^{4}$ \\ Kyung S. Lee, ${ }^{5}$ and Yong C. Lee ${ }^{5}$ \\ ${ }^{1}$ Department of Pharmacology, Institute of Cardiovascular Research, \\ ${ }^{2}$ Department of Anatomy, \\ ${ }^{3}$ Department of Pediatrics, \\ ${ }^{4}$ Department of Biochemistry, and \\ ${ }^{5}$ Department of Internal Medicine, Research Center for Allergic Immune Diseases, Chonbuk National University Medical \\ School, Chonju, South Korea
}

\begin{abstract}
Phosphatase and tensin homologue deleted on chromosome ten (PTEN) is part of a complex signaling system that affects a variety of important cell functions. PTEN blocks the action of PI3K by dephosphorylating the signaling lipid phosphatidylinositol 3,4,5-triphosphate. We have used a mouse model for asthma to determine the effect of PI3K inhibitors and PTEN on allergen-induced bronchial inflammation and airway hyperresponsiveness. PI3K activity increased significantly after allergen challenge. PTEN protein expression and PTEN activity were decreased in OVA-induced asthma. Immunoreactive PTEN localized in epithelial layers around the bronchioles in control mice. However, this immunoreactive PTEN dramatically disappeared in allergen-induced asthmatic lungs. The increased IL-4, IL-5, and eosinophil cationic protein levels in bronchoalveolar lavage fluids after OVA inhalation were significantly reduced by the intratracheal administration of PI3K inhibitors or adenoviruses carrying PTEN cDNA (AdPTEN). Intratracheal administration of PI3K inhibitors or AdPTEN remarkably reduced bronchial inflammation and airway hyperresponsiveness. These findings indicate that PTEN may play a pivotal role in the pathogenesis of the asthma phenotype.
\end{abstract}

J. Clin. Invest. 111:1083-1092 (2003). doi:10.1172/JCI200316440.

\section{Introduction}

Bronchial asthma is a chronic inflammatory disease of the airways characterized by airway eosinophilia, goblet cell hyperplasia with mucus hypersecretion, and hyperresponsiveness to inhaled allergens and to nonspecific stimuli (1). Eosinophil response appears to be a critical feature in asthma. Eosinophil accumulation and subsequent activation in bronchial tissues play critical roles in the pathophysiology of bronchial asthma (2). Many inflammatory mediators attract and activate eosinophils via signal transduction pathways involving the enzyme PI3K. Several studies using wortmannin, a specific inhibitor of PI3K, have revealed the involvement of PI3K in the biochemical transduction of activation signals generated by many inflammatory mediators in eosinophils (3-7). Wortmannin plays a

Received for publication July 17, 2002, and accepted in revised form February 4, 2003.

Address correspondence to: Yong Chul Lee, Department of Internal Medicine, Chonbuk National University Medical School, 634-18 Keumamdong, Chonju 561-712, South Korea.

Phone: 82-63-250-1664; Fax: 82-63-254-1609;

E-mail: leeyc@moak.chonbuk.ac.kr.

Conflict of interest: The authors have declared that no conflict of interest exists.

Nonstandard abbreviations used: phosphatase and tensin homologue deleted on chromosome ten (PTEN);

phosphatidylinositol 3,4,5-triphosphate (PIP3); adenovirus (Ad); bronchoalveolar lavage (BAL); eosinophil cationic protein (ECP); enhanced pause (Penh); phosphorylated Akt (p-Akt). role in preventing the development of airway hyperresponsiveness by preventing either eosinophil infiltration of bronchial tissues or eosinophil activation on arrival. Phosphatase and tensin homologue deleted on chromosome ten (PTEN) functions primarily as a lipid phosphatase to regulate crucial signal transduction pathways (8). PTEN has been implicated in regulating cell survival signaling through the PI3K/Akt pathway. PTEN blocks the action of PI3K by dephosphorylating the signal lipid phosphatidylinositol 3,4,5-triphosphate (PIP3). PIP3, produced by PI3K following activation by receptor tyrosine kinases, activated Ras, or $G$ proteins, leads to the stimulation of several downstream targets, including the serine/ threonine protein kinase Akt (also known as protein kinase B) (9). PTEN is known to be critically important during embryonic development and in mature organisms. Studies of its functions are providing novel insights into the regulation of apoptosis, migration, and tumor progression. PTEN appears to serve as a hub or switchpoint linking complex signaling pathways. However, no data are available on the role of PTEN in bronchial asthma.

In the present study we used a murine model of asthma to examine the involvement of PTEN in the pathogenesis of bronchial asthma. In addition, we found evidence that specific inhibitors of PI3K or adenovirus (Ad) gene transfer vector expressing a PTEN cDNA inhibit airway inflammation and airway hyperresponsiveness. 


\section{Methods}

Animals and experimental protocol. Female BALB/c mice, 8-10 weeks of age and free of murine specific pathogens, were obtained from the Korean Research Institute of Chemistry Technology (Daejon, Korea). The mice were housed throughout the experiments in a laminar flow cabinet and were maintained on standard laboratory chow ad libitum. All experimental animals used in this study were treated according to guidelines approved by the Institutional Animal Care and Use Committee of the Chonbuk National University Medical School. Mice were sensitized on days 1 and 14 by intraperitoneal injection of $20 \mu \mathrm{g}$ OVA (SigmaAldrich, St. Louis, Missouri, USA) emulsified in $1 \mathrm{mg}$ of aluminum hydroxide (Pierce Chemical Co., Rockford, Illinois, USA) in a total volume of $200 \mu \mathrm{l}$ (Figure 1). On days 21,22 , and 23 after the initial sensitization, the mice were challenged for 30 minutes with an aerosol of $1 \%(\mathrm{wt} / \mathrm{vol}$ ) OVA in saline (or with saline as a control) using an ultrasonic nebulizer (NE-U12; Omron Corp., Tokyo, Japan). Bronchoalveolar lavage (BAL) was performed 72 hours after the last challenge. At the time of lavage, the mice (six mice in each group) were sacrificed with an overdose of sodium pentobarbitone (pentobarbital sodium, $100 \mathrm{mg} / \mathrm{kg}$ body weight, administered intraperitoneally). The chest cavity was exposed to allow for expansion, after which the trachea was carefully intubated and the catheter secured with ligatures. Prewarmed $0.9 \% \mathrm{NaCl}$ solution was slowly infused into the lungs and withdrawn. The aliquots were pooled and stored at $4^{\circ} \mathrm{C}$. Part of each pool was then centrifuged and the supernatants were kept at $-70^{\circ} \mathrm{C}$ until use. Total cell numbers were counted with a hemocytometer. Smears of BAL cells were prepared by cytospin (Shandon Scientific Ltd., Cheshire, United Kingdom). The smears were stained with Diff-Quik solution (Dade Diagnostics of Puerto Rico Inc., Aguada, Puerto Rico) in order to examine the cell differentials. Two independent, blinded investigators counted the cells using a microscope. Approximately 400 cells were counted in each of four different random locations. Variation of results between investigators was less than $5 \%$. The mean of the values from the two investigators was used for each cell count.

Measurement of inducible PI3K enzyme activity. Inducible phosphotyrosine-associated PI3K activity was determined. Whole lung tissues from the mice were homogenized in $1 \mathrm{ml}$ of a solution containing $1 \%$ Nonidet P-40, $50 \mathrm{mM}$ Tris, $100 \mathrm{mM} \mathrm{NaCl}, 50 \mathrm{mM} \mathrm{NaF}, 10 \mathrm{mM}$ tetrasodium pyrophosphate, $2 \mathrm{mM}$ orthovanadate, 2.5 $\mathrm{mM}$ benzamidine, $1 \mathrm{mM}$ PMSF, and $1 \mu \mathrm{M}$ DTT, as previously reported $(10,11)$. Clarified lysates obtained by centrifugation at $14,000 \mathrm{~g}$ for 20 minutes were added to a mixture containing anti-phosphotyrosine antibody 4G10 (Upstate Biotechnology Inc., Lake Placid, New York, USA) and $20 \mu \mathrm{l}$ of agarose beads (SigmaAldrich). The anti-phosphotyrosine complexes were washed twice with buffer A (1\% Nonidet P-40, $1 \mathrm{mM}$ DTT, and $10 \mathrm{mM}$ Tris, $\mathrm{pH}$ 7.4), twice with buffer B (0.5
$\mathrm{M} \mathrm{LiCl}, 1 \mathrm{mM}$ DTT, and $100 \mathrm{mM}$ Tris, $\mathrm{pH}$ 7.4), and then twice with buffer $\mathrm{C}(10 \mathrm{mM} \mathrm{NaCl}, 1 \mathrm{mM}$ DTT, and $10 \mathrm{mM}$ Tris, $\mathrm{pH}$ 7.4). The lipid kinase reaction was initiated by addition of $60 \mu \mathrm{l}$ sonicated L- $\alpha$-phosphatidylinositol $(0.33 \mathrm{mg} / \mathrm{ml})$ in a kinase assay buffer containing 20 mM HEPES (pH 7.1), 0.4 mM EGTA, 0.4 $\mathrm{mM} \mathrm{NaPO}_{4}, 48 \mu \mathrm{M}\left[\gamma^{3}{ }^{32} \mathrm{P}\right] \mathrm{ATP}(2.1 \mu \mathrm{Ci} / \mathrm{mmol}$; Amersham Biosciences, Buckinghamshire, United Kingdom), and $10 \mathrm{mM} \mathrm{MgCl}$. The reactions were performed at room temperature for 15 minutes and terminated by addition of $15 \mu \mathrm{l}$ of $4 \mathrm{~N} \mathrm{HCl}$. Lipids were extracted by addition of $200 \mu \mathrm{l}$ of a solution containing equal volumes of chloroform and methanol, followed by vortexing and centrifugation at $14,000 \mathrm{~g}$ for 10 minutes. The chloroform-containing lipid phase was then re-extracted with $150 \mu \mathrm{l}$ of an equal-volume mixture of $0.15 \mathrm{~N} \mathrm{HCl}$ and methanol, followed by vortexing and centrifugation at $14,000 \mathrm{~g}$ for 10 minutes. Thirty microliters of the chloroform phase was resolved by TLC using a running solution consisting of chloroform, methanol, and ammonium hydroxide (volume ratio, 75:58:17). Detection of phosphorylated lipid was performed by PhosphorImager analysis (Molecular Dynamics, Sunnyvale, California, USA).

Vectors. The E1/E3-deleted replication-deficient recombinant Ad was made using the AdEasy system (Quantum Biotechnologies, Montreal, Quebec, Canada) described by He et al. (12). KpnI-XhoI restriction fragments from pcDNA3/wild-type PTEN cDNA were ligated into KpnIXhoI-digested pShuttleCMV, as previously described (13). To create AdLacZ, a SalI-NotI restriction fragment from pcDNA3.1/LacZ (Invitrogen Corp., San Diego, California, USA) was ligated to SalI-NotI-digested pShuttleCMV. Recombination into the pAdEasy viral backbone was accomplished in bacteria (E. coli strain BJ5183, which is recombination deficient) according to the manufacturer's instructions. The recombination was verified and the adenoviral recombinant DNA was transferred to a regular strain of $E$. coli (DH5 $\alpha$ ), which generates far greater yields of DNA. Recombinant pAdEasy plasmids containing CMV-cDNA inserts were purified over QIAGEN columns (QIAGEN Inc., Valencia, California, USA), and $5 \mu \mathrm{g}$ of PacI-digested DNA was used to transfect QBI-293A cells using the calcium phosphate method (Promega Corp., Madison, Wisconsin, USA). Cells were seeded at $2 \times 10^{6}$ cells per 150 -mm culture dish 24 hours prior to transfection. Lysis of transfected cells, indicating adenoviral growth, occurred within 4 days. Following amplification, lysates containing clonal recombinant Ad were prepared from 150-mm culture dishes and purified by $\mathrm{CsCl}$ gradient centrifugation. Recovered virus was aliquoted and stored at $-20^{\circ} \mathrm{C}$ in $5 \mathrm{mM}$ Tris ( $\mathrm{pH}$ 8.0) buffer containing $50 \mathrm{mM} \mathrm{NaCl}, 0.05 \% \mathrm{BSA}$, and $25 \%$ glycerol. Virus was titrated by serial dilution infection of QBI-293A cells and plaques were counted under an overlay of $0.3 \%$ agarose, $10 \% \mathrm{FBS}$, and $1 \times$ DMEM.

Administration of wortmannin, LY-294002, or Ad vectors. Wortmannin $(100 \mu \mathrm{g} / \mathrm{kg}$ body wt/day; CalbiochemNovabiochem Corp., San Diego, California, USA) or 


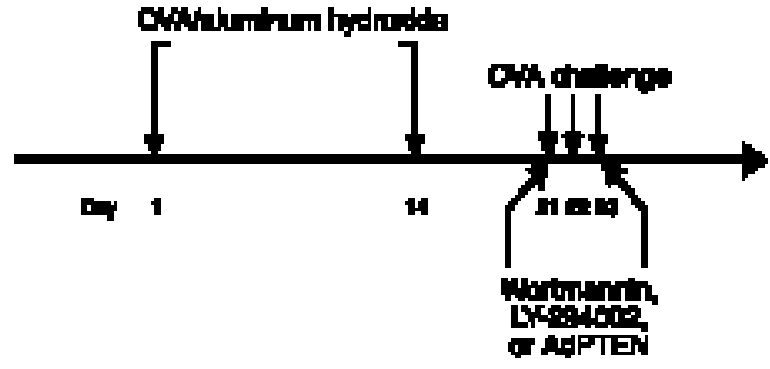

Figure 1

Schematic diagram of the experimental protocol. Mice were sensitized on days 1 and 14 by intraperitoneal injection of OVA emulsified in 1 $\mathrm{mg}$ aluminum hydroxide. On days 21,22 , and 23 after the initial sensitization, the mice were challenged for 30 minutes with an aerosol of $1 \%(\mathrm{wt} / \mathrm{vol}$ ) OVA in saline (or with saline as a control) using an ultrasonic nebulizer. Wortmannin, LY-294002, or Ad vector was administered intratracheally two times to each treated animal, once on day 21 ( 1 hour before the first airway challenge with OVA) and the second time on day 23 ( 3 hours after the last airway challenge with OVA).

LY-294002 [2-(4-morpholinyl)-8-phenyl-4H-1-benzopyran-4-one] $(1.5 \mathrm{mg} / \mathrm{kg}$ body wt/day; BIOMOL Research Laboratories Inc., Plymouth Meeting, Pennsylvania, USA) dissolved in DMSO and diluted with $0.9 \% \mathrm{NaCl}$ was administered in a volume of $50 \mu \mathrm{l}$. Wortmannin, LY-294002, or Ad vector was administered intratracheally two times to each treated animal, once on day 21 (1 hour before the first airway challenge with OVA) and the second time on day 23 ( 3 hours after the last airway challenge with OVA) (Figure 1). The vehicle was $0.9 \% \mathrm{NaCl}$ containing DMSO. Ad vectors ( $10^{9}$ plaque-forming units) were administered intratracheally two times to each animal under the same administration schedule described above (Figure 1).

Isolation of BAL fluid eosinophils. Eosinophils were purified as previously described (14). Briefly, BAL cells were centrifuged at $1,500 \mathrm{~g}$ at $4^{\circ} \mathrm{C}$ for 10 minutes. The supernatant was kept at $-70^{\circ} \mathrm{C}$ and the pellets were resuspended in RPMI 1640 (Invitrogen Corp.). The suspended cells were pooled and layered onto a discontinuous Percoll gradient consisting of $2 \mathrm{ml}$ of $72 \%$ and $3 \mathrm{ml}$ of $56 \%$ Percoll. After centrifugation at $2,200 \mathrm{~g}$ at $4^{\circ} \mathrm{C}$ for 30 minutes, eosinophils were carefully collected from the interface between the Percoll bands and washed twice with RPMI 1640. The final cell pellets were resuspended in PBS. Eosinophil purity was determined by staining cytospin preparations (Shandon Scientific Ltd.) with Diff-Quik. In all preparations, purity of eosinophils was $85-95 \%$.

Measurement of cytokines. Levels of IL-4 and IL-5 were quantified in the supernatants of BAL fluids by enzyme immunoassays according to the manufacturer's protocol (Endogen Inc., Woburn, Massachusetts, USA). Sensitivity for assays was $5 \mathrm{pg} / \mathrm{ml}$.

Measurement of eosinophil cationic protein. Eosinophil cationic protein (ECP) was measured in the supernatants of BAL fluids. Levels of ECP were quantified by fluoroenzyme immunoassay according to the manufacturer's protocol (UniCAP ECP; Pharmacia \& Upjohn Diagnostics AB, Uppsala, Sweden). Sensitivity for the assay was $2 \mathrm{ng} / \mathrm{ml}$.

Determination of airway responsiveness to methacholine. Airway responsiveness was measured in unrestrained, conscious mice 3 days after the last challenge, as previously described $(15,16)$. Mice were placed in a barometric plethysmographic chamber (All Medicus Co., Seoul, Korea), and baseline readings were taken and averaged for 3 minutes. Aerosolized methacholine in increasing concentrations (from 2.5 to $50 \mathrm{mg} / \mathrm{ml}$ ) was nebulized through an inlet of the main chamber for 3 minutes. Readings were taken and averaged for $3 \mathrm{~min}-$ utes after each nebulization and enhanced pause (Penh) was determined. Penh, calculated as (expiratory time/relaxation time -1$) \times($ peak expiratory flow/peak inspiratory flow) according to the manufacturers' protocol, is a dimensionless value that represents a function of the proportion of maximal expiratory to maximal inspiratory box pressure signals and a function of the timing of expiration. Penh is used as a measure of airway responsiveness to methacholine. Results are expressed as the percentage increase of Penh following challenge with each concentration of methacholine, where the baseline Penh (after saline challenge) is expressed as $100 \%$. Penh values averaged for 3 minutes after each nebulization were evaluated.

Measurement of PTEN activity. PTEN activities were measured using the PTEN malachite green assay kit according to the protocol of the manufacturer (Upstate Biotechnology Inc.).

Isolation and primary culture of murine tracheal epithelial cells. Murine tracheal epithelial cells were isolated under sterile conditions. The trachea proximal to the

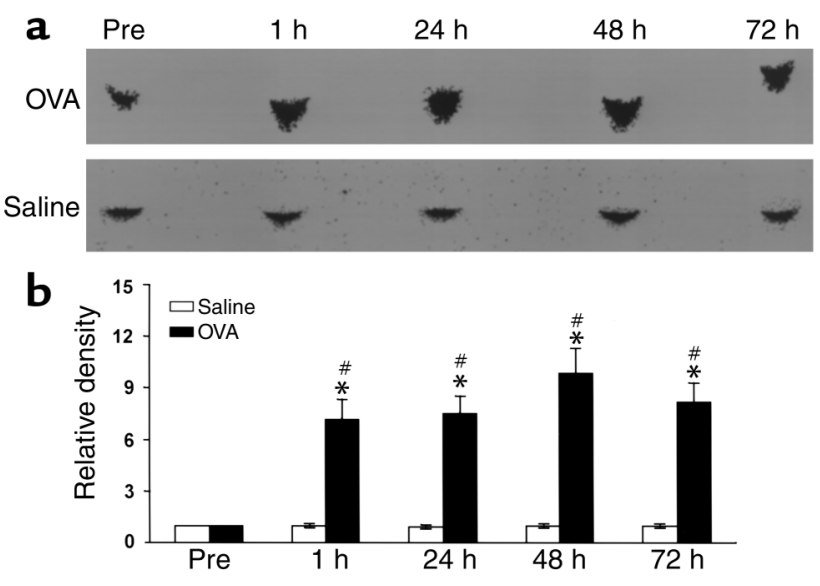

Figure 2

Inducible PI3K enzyme activity in lung tissues of OVA-sensitized and challenged mice. (a) PI3K activity was measured in lung tissues from sensitized mice challenged with OVA (upper panel) or with saline (lower panel). (b) Results of densitometric analysis are presented as the relative ratio of induction of $\mathrm{PI} 3 \mathrm{~K}$ before and after the challenge. The $\mathrm{PI} 3 \mathrm{~K}$ activity in the lung tissue of preinhalation mice is arbitrarily presented as 1 . Data represent mean \pm SD from six independent experiments. Pre, before challenge; $1,24,48$, and 72 hours are time periods after the last challenge. ${ }^{*} P<0.05$ vs. Pre; ${ }^{\#} P<0.05$ vs. saline inhalation. 
a

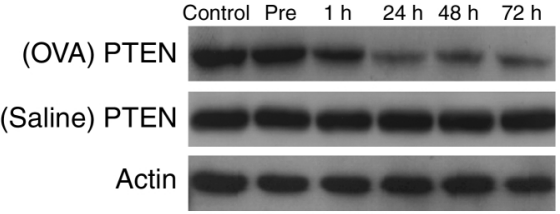

b

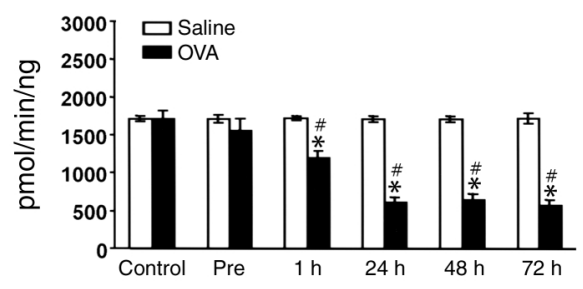

Figure 3

Western blot analyses of PTEN protein and PTEN activity in lung tissues of OVA-sensitized and -challenged mice. (a) Western blot analyses of PTEN protein. Sampling was performed in lung tissues from sensitized mice challenged with OVA or saline. The Western blot was probed with an anti-PTEN antibody and reprobed with an anti-actin antibody to verify equal loading of protein in each lane. Results were similar in six independent experiments. (b) PTEN activity was measured in lung tissues from sensitized mice challenged with OVA or with saline. Data are presented as mean \pm SD from six independent experiments. ${ }^{*} P<0.05$ vs. Pre; ${ }^{\#} P<0.05$ vs. saline inhalation. Control, no treatment.

bronchial bifurcation was excised and adherent adipose tissue was removed. The trachea was opened longitudinally and cut into three pieces. The isolated tracheas were incubated in DMEM containing 0.1\% protease overnight at $4^{\circ} \mathrm{C}$. Following tissue digestion, FBS (10\% final concentration) was added to the medium to deactivate enzymes, undigested fragments of tissue were removed, and tracheal epithelial cells were harvested by centrifugation at $100 \mathrm{~g}$ for 5 minutes. The epithelial cells were seeded onto $35-\mathrm{mm}$ collagencoated dishes for submerged culture. The growth medium DMEM/F-12 (Sigma-Aldrich) containing $10 \%$ FBS, penicillin, streptomycin, and amphotericin $B$ was supplemented with insulin, transferrin, hydrocortisone, phosphoethanolamine, cholera toxin, ethanolamine, bovine pituitary extract, and BSA. The cells were maintained in a humidified $5 \% \mathrm{CO}_{2}$ incubator at $37^{\circ} \mathrm{C}$ until they adhered.

Histology, immunohistochemistry, and immunocytochemistry. At 72 hours after the last challenge, mice were sacrificed and the lungs and trachea were filled intratracheally with a fixative ( $0.8 \%$ formalin, $4 \%$ acetic acid) using a ligature around the trachea. Lungs were removed and lung tissues were fixed with $10 \%$ (vol/vol) neutral buffered formalin. The specimens were dehydrated and embedded in paraffin. For histologic examination, $4-\mu \mathrm{m}$ sections of fixed embedded tissues were cut on a Leica model 2165 rotary microtome (Leica Microsystems Nussloch GmbH, Nussloch, Germany), placed on glass slides, deparaffinized, and stained sequentially with hematoxylin 2 and eosin Y (RichardAllan Scientific, Kalamazoo, Michigan, USA). Inflammation was scored by three independent blinded investigators. The degree of peribronchial and perivascular inflammation was evaluated on a subjective scale of $0-3$, as described elsewhere (17). A value of 0 was assigned when no inflammation was detectable, a value of 1 was assigned for occasional cuffing with inflammatory cells, a value of 2 was assigned for most bronchi or vessels surrounded by a thin layer (one to five cells thick) of inflammatory cells, and a value of 3 was given when most bronchi or vessels were surrounded by a thick layer (more than five cells thick) of inflammatory cells. Total lung inflammation was defined as the average of the peribronchial and perivascular inflammation scores.

For immunohistochemistry and immunocytochemistry of PTEN, the deparaffinized $4-\mu \mathrm{m}$ sections or the cytocentrifuge preparations of BAL cells, BAL eosinophils, or tracheal epithelial cells were incubated sequentially in accordance with instructions for the RTU Vectastain Universal Quick Kit from Vector Laboratories Inc. (Burlingame, California, USA). Briefly, the slides were incubated in Endo/Blocker (Biomeda Corp., Foster City, California, USA) for 5 minutes and in pepsin solution for 4 minutes at $40^{\circ} \mathrm{C}$. The slides were incubated in normal horse serum for 15 minutes at room temperature. The slides were probed with an affinity-purified rabbit polyclonal PTEN IgG (Santa Cruz Biotechnology Inc., Santa Cruz, California, USA) overnight at $4^{\circ} \mathrm{C}$, and were incubated with prediluted biotinylated panspecific IgG for 10 minutes. The slides were incubated with HRP-conjugated streptavidin for 5 minutes and then in 3-amino-9-ethylcarbazole substrate mixtures for peroxidase for 12 minutes. For the control, sections of lung tissue, BAL cells, BAL eosinophils, or tracheal epithelial cells prepared from mice were treated without the primary antibody under the same conditions. After immunostaining, the slides were counterstained for 1 minute with Gill's hematoxylin in $20 \%$ ethylene glycol and then mounted with Aqueous Mounting Medium from InnoGenex (San Ramon, California, USA) and photomicrographed (Vanox T; Olympus Optical Co., Tokyo, Japan).

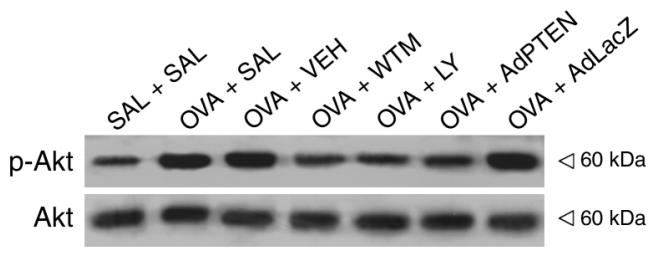

\section{Figure 4}

Effect of wortmannin, LY-294002, or AdPTEN on p-Akt and Akt protein expression in lung tissues of OVA-sensitized and -challenged mice. $\mathrm{p}$-Akt and Akt protein expression were measured 72 hours after the last challenge in saline-inhaled mice administered saline intratracheal ly (SAL + SAL), OVA-inhaled mice administered saline (OVA $+\mathrm{SAL})$, OVA-inhaled mice administered drug vehicle (OVA + VEH), OVA-inhaled mice administered wortmannin (OVA + WTM), OVAinhaled mice administered LY-294002 (OVA + LY), OVA-inhaled mice administered AdPTEN (OVA + AdPTEN), and OVA-inhaled mice administered AdLacZ (OVA + AdLacZ). Results were similar in six independent experiments. 


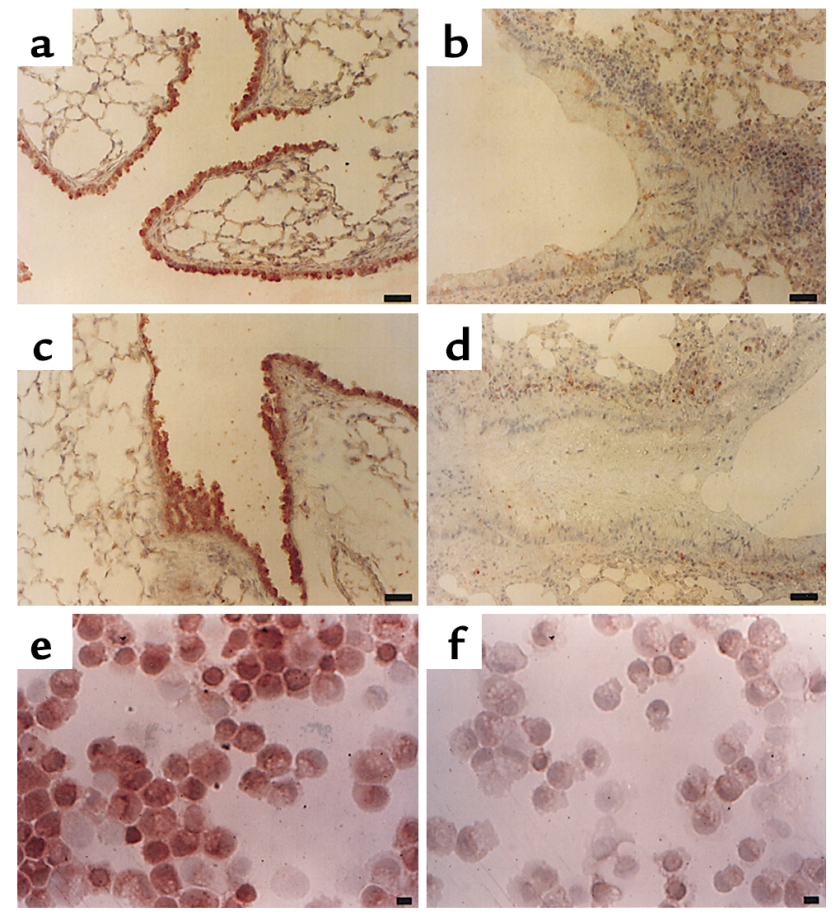

Western blot analysis. Lung tissues were homogenized in the presence of protease inhibitors to obtain extracts of lung proteins. Protein concentrations were determined using Bradford reagent (Bio-Rad Laboratories Inc., Hercules, California, USA). Samples ( $30 \mu \mathrm{g}$ protein per lane) were loaded on a $12 \%$ SDS-PAGE gel. After electrophoresis at $120 \mathrm{~V}$ for 90 minutes, separated proteins were transferred to PVDF membranes (Amersham Pharmacia Biotech, Piscataway, New Jersey, USA) by the wet transfer method ( $250 \mathrm{~mA}$ for 90 minutes). Nonspecific sites were blocked with $5 \%$ nonfat milk in TBST buffer $(25 \mathrm{mM}$ Tris, pH 7.5, $150 \mathrm{mM} \mathrm{NaCl}$, and $0.1 \%$ Tween 20 ) for 1 hour, and the blots were then incubated overnight at $4{ }^{\circ} \mathrm{C}$ with anti-PTEN antibody (Santa Cruz Biotechnology Inc.) diluted 1:800 or with antibody against Akt or phosphorylated Akt (p-Akt; Cell Signaling Technology Inc., Beverly, Massachusetts, USA) diluted 1:1,000. Anti-rabbit HRP-conjugated IgG was used to detect binding of antibodies. Binding of the specific antibodies was visualized by exposing to

\section{Figure 6}

Localization of immunoreactive PTEN in BAL fluids of OVA-sensitized and -challenged mice. Sampling was performed 72 hours after the last challenge in BAL fluids from sensitized mice challenged with saline (a, e, and $\mathbf{f}$ ), sensitized mice challenged with OVA (b), OVAinhaled mice administered AdPTEN (c), and OVA-inhaled mice administered AdLacZ (d). Representative light microscopy shows PTEN-positive cells in the BAL fluids $(\mathbf{a}, \mathbf{b}, \mathbf{c}, \mathbf{d}$, and $\mathbf{e})$; the brown color indicates PTEN-positive cells. (f) To examine the cell differentials in BAL cells prepared from the control mice, the slides used for the detection of PTEN (e) were destained with $70 \%$ ethyl alcohol. The smears of BAL cells were stained with Diff-Quik solution and viewed under a light microscope. The arrow indicates a macrophage; the arrowhead indicates a lymphocyte. Bars indicate $10 \mu \mathrm{m}$.

\section{Figure 5}

Localization of immunoreactive PTEN in lung tissues and tracheal epithelial cells of OVA-sensitized and -challenged mice. Sampling was performed 72 hours after the last challenge in lung tissues and tracheal epithelial cells from sensitized mice challenged with saline ( $\mathbf{a}$ and $\mathbf{e}$ ), from sensitized mice challenged with OVA $(\mathbf{b}$ and $\mathbf{f})$, from OVA-inhaled mice administered AdPTEN (c), and from OVA-inhaled mice administered AdLacZ (d). Representative light microscopy shows PTEN-positive cells in the bronchioles $(\mathbf{a}, \mathbf{b}, \mathbf{c}$, and $\mathbf{d}$; the brown color indicates PTEN-positive cells) and in the tracheal epithelial cells (e and $\mathbf{f}$; the dark brown color indicates PTEN-positive cells). Bars indicate scale of $50 \mu \mathrm{m}(\mathbf{a}, \mathbf{b}, \mathbf{c}$, and $\mathbf{d})$ or $10 \mu \mathrm{m}$ (e and $\mathbf{f})$.

photographic film after treating with ECL system reagents (Amersham Pharmacia Biotech).

Assessment of LacZ gene expression in airways. To assess the distribution of $L a c Z$ gene expression by Ad vectors in airways, $\beta$-gal staining was performed in lung tissues and BAL fluid eosinophils. LacZ-expressing cells were detected in situ as blue-stained cells by $\beta$-gal staining (18). Sampling was performed 24, 48, and 72 hours after the last airway challenge with OVA. Lungs were inflated with $2 \%$ paraformaldehyde in PBS for 1 hour and then sectioned. BAL fluid eosinophils were also fixed with $2 \%$ paraformaldehyde in PBS for 1 hour. After fixation, lung tissues and BAL fluid eosinophils were washed twice with cold PBS containing $1 \mathrm{mM} \mathrm{MgCl} 2$ and then stained with $\beta$-gal for 24 hours at $37^{\circ} \mathrm{C}$. Tissue sections were counterstained with $\mathrm{H} \& \mathrm{E}$ after $\beta$-gal staining.

Statistical analysis. Data are expressed as mean \pm SD. Statistical comparisons were performed using oneway ANOVA followed by the Fisher test. Significant differences between groups were determined using the unpaired Student $t$ test. Statistical significance was set at $P<0.05$.
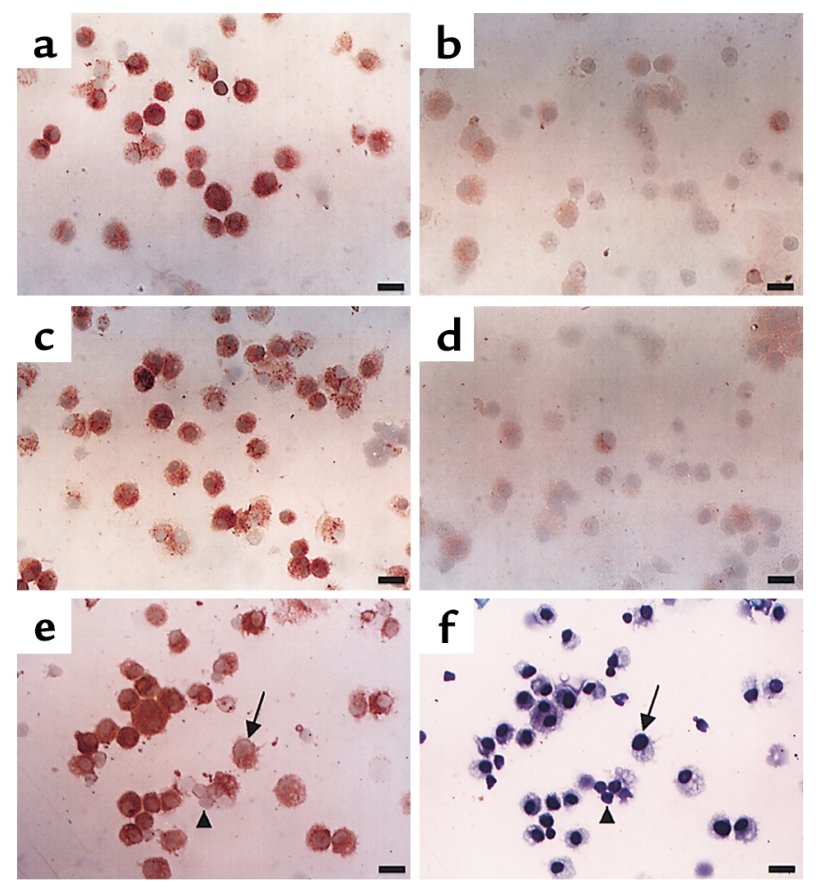

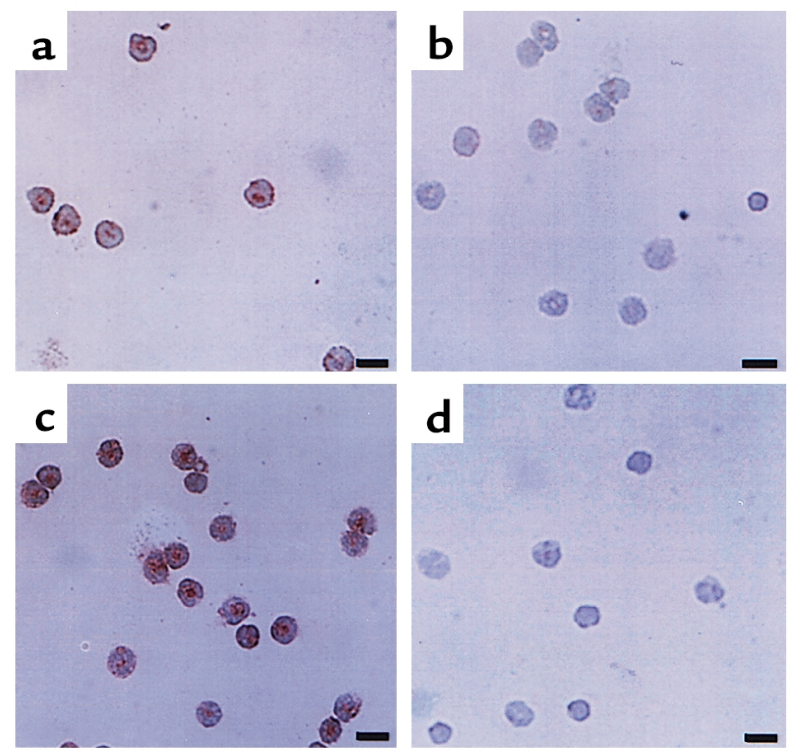

\section{Figure 7}

Representative photomicrographs showing localization of immunoreactive PTEN in BAL fluid eosinophils of OVA-sensitized and -challenged mice. Sampling was performed 72 hours after the last challenge in BAL fluids from sensitized mice challenged with saline (a), from sensitized mice challenged with OVA (b), from OVA-inhaled mice administered $\operatorname{AdPTEN}(\mathbf{c})$, and from OVA-inhaled mice administered AdLacZ (d). Representative light microscopy of PTEN-positive cells in the BAL eosinophils; the brown color indicates PTEN-positive cells. Bars indicate $10 \mu \mathrm{m}$.

\section{Results}

PI3K activity increased in OVA-induced asthma. In OVAexposed mice, PI3K activity had increased approximately 7.2-, 7.5-, 9.8-, and 8.2-fold at 1, 24, 48, and 72 hours after OVA inhalation, respectively, compared with the prechallenge period (Figure 2). In contrast, no significant changes in PI3K activity were observed after saline inhalation.

PTEN protein expression and PTEN activity decreased in OVA-induced asthma. Western blot analysis revealed that PTEN protein levels were decreased after challenge with OVA compared with levels before OVA inhalation or in the control group (Figure 3a). In contrast, no significant changes in the PTEN protein level were observed after saline inhalation. Consistent with these results, PTEN enzyme assays revealed that PTEN activity was significantly decreased at $1,24,48$, and 72 hours after OVA inhalation compared with levels before OVA inhalation or in the control group (Figure 3b). On the other hand, no significant changes in PTEN activity were observed after saline inhalation.

Determination of Akt phosphorylation. To support the contention that the effects of wortmannin, LY-294002, or AdPTEN on allergen-induced bronchial inflammation and airway hyperresponsiveness were specifically directed through the PI3K pathway, we used Western blotting to determine Akt phosphorylation. Levels of pAkt protein in the lung tissues were increased at 72 hours after OVA inhalation compared with levels in the control mice (Figure 4). However, no significant changes in Akt protein levels were observed in any of the groups tested. The increased p-Akt but not Akt protein levels in lung tissues at 72 hours after OVA inhalation were significantly reduced by the intratracheal administration of wortmannin, LY-294002, or AdPTEN.

Localization of immunoreactive PTEN in lung tissues, tracheal epithelial cells, and BAL fluids of OVA-induced asthma. Immunoreactive PTEN localized in epithelial layers around the bronchioles in control mice (Figure 5a). This immunoreactive PTEN dramatically disappeared in allergen-induced asthmatic lungs (Figure 5b). Interestingly, intratracheal administration of AdPTEN restored the immunoreactive PTEN expression in allergen-induced asthmatic mouse lungs (Figure $5 \mathrm{c}$ ), whereas AdLacZ did not (Figure 5d). The expression of PTEN in lung epithelial cells after isolation and primary culture of murine tracheal epithelial cells was observed. Immunocytologic analysis showed localization of immunoreactive PTEN in the tracheal epithelial cells from control mice (Figure 5e). However, immunoreactive PTEN was markedly reduced in tracheal epithelial cells from OVA-exposed mice (Figure 5f).

Immunocytologic analysis of BAL fluids showed localization of immunoreactive PTEN in the BAL cells from control mice (Figure 6, a and e). However, immunoreactive PTEN was markedly reduced in precipitated cells from OVA-exposed mice (Figure 6b).
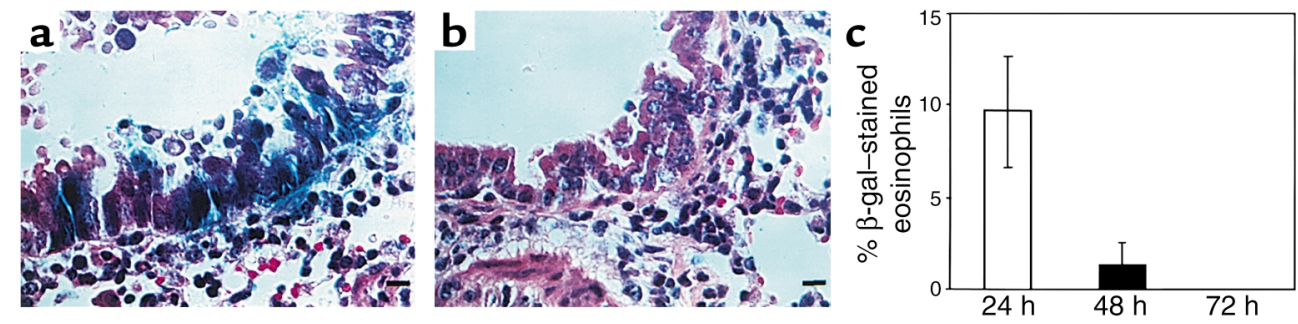

\section{Figure 8}

Localization of immunoreactive $\beta$-gal in lung tissues and BAL fluid eosinophils of OVA-sensitized and -challenged mice. Sampling was performed 72 hours after the last challenge in lung tissues from OVA-inhaled mice administered AdLacZ (a) and sensitized mice challenged with OVA (b). Blue-stained cells were considered to express the LacZ gene. Bars indicate $10 \mu \mathrm{m}$ (a and $\mathbf{b})$. Percentage of $\beta$-gal-stained cells in BAL fluid eosinophils (c). Sampling was performed at 24,48 , and 72 hours after the last challenge in BAL fluids from OVA-inhaled mice administered AdLacZ. Data represent mean \pm SD from six independent experiments. 

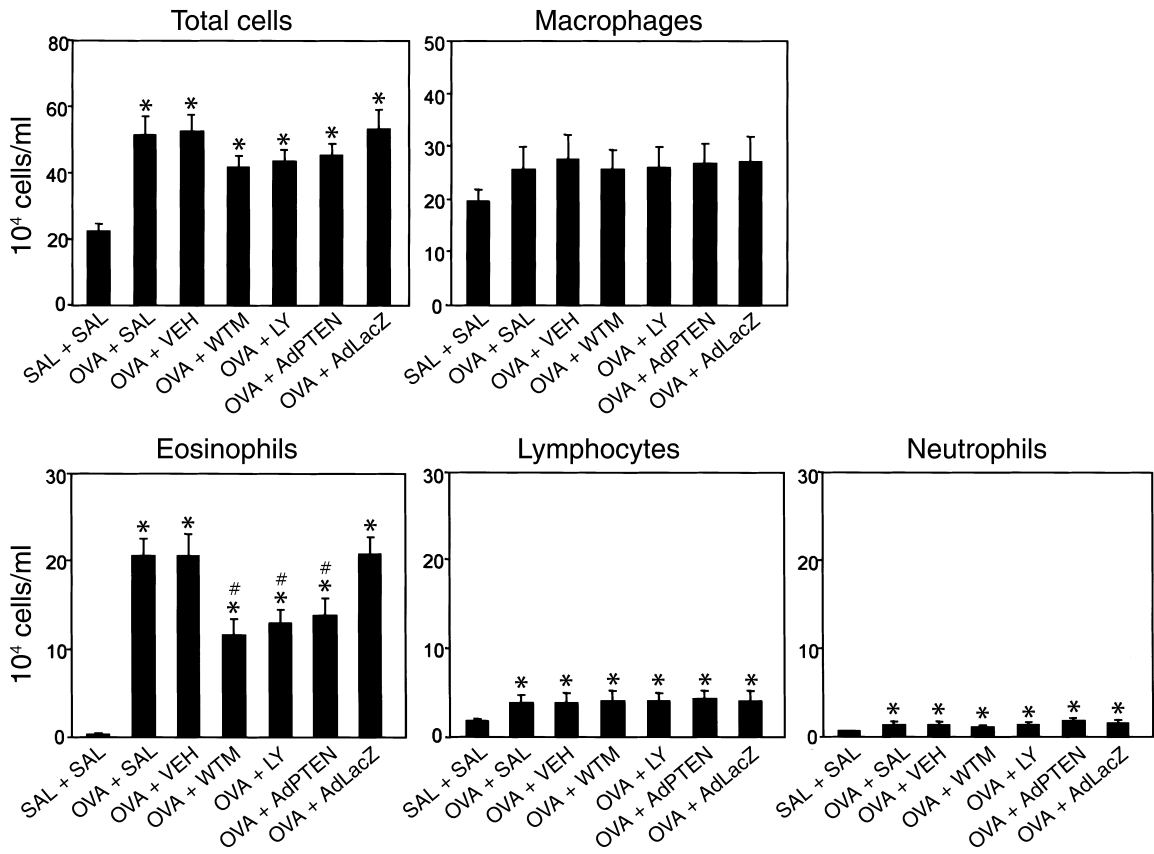

\section{Figure 9}

Effect of wortmannin, LY-294002, or AdPTEN on total and differential cellular components of BAL. Shown are numbers of each cellular component of BAL from mice treated as described in Figure 4 legend, counted 72 hours after the last challenge. Bars represent mean \pm SD from six independent experiments. ${ }^{*} P<0.05$ vs. SAL + SAL; ${ }^{P} P<0.05$ vs. OVA + SAL and OVA + AdLacZ.
Intratracheal administration of AdPTEN restored immunoreactive PTEN expression in BAL cells from the allergen-induced asthmatic mice (Figure $6 \mathrm{c}$ ), whereas AdLacZ did not (Figure 6d).

To examine the cell differentials present in BAL cells, the slides used for the detection of PTEN were destained with $70 \%$ ethyl alcohol. The smears of BAL cells were stained with Diff-Quik solution. Immunoreactive PTEN was localized on macrophages and lymphocytes (Figure 6, e and f). Treatment with OVA resulted in reduction of immunoreactive PTEN on the macrophages and lymphocytes in the BAL fluid (Figure 6b) compared with that from the control mice.

In addition, the expression of PTEN in BAL fluid eosinophils isolated by Percoll gradient centrifugation was examined by immunocytology. Localization of immunoreactive PTEN was observed in BAL fluid eosinophils from the control mice (Figure 7a). However, immunoreactive PTEN was markedly reduced in eosinophils from OVA-exposed mice (Figure 7b). Intratracheal administration of AdPTEN restored the immunoreactive PTEN expression in BAL fluid eosinophils from the allergen-induced asthmatic mice (Figure 7c), and additional AdLacZ treatment did not affect immunoreactivity in OVA-exposed mice (Figure $7 d)$. We determined which cells were being infected by Ad PTEN by $\beta$-gal staining of AdLacZ-infected cells. Immunoreactive $\beta$-gal localized in epithelial layers around bronchioles prepared 24-72 hours after the last airway challenge with OVA. Cells staining positive for $\beta$-gal were also observed in eosinophils in BAL fluid obtained from the OVA-challenged airway. Percentages

\section{Figure 10}

Effect of wortmannin, LY-294002, or AdPTEN in lung tissues of OVA-sensitized and -challenged mice. Representative H\&E-stained sections of the lungs. Sampling was performed 72 hours after the last challenge in saline-inhaled mice administered saline (a), OVA-inhaled mice administered saline (b), OVA-inhaled mice administered wortmannin (c), OVA-inhaled mice administered LY-294002 (d), OVA-inhaled mice administered $\operatorname{AdPTEN}(\mathbf{e})$, and OVA-inhaled mice administered AdLacZ (f). Bars indicate scale of $50 \mu \mathrm{m}$.
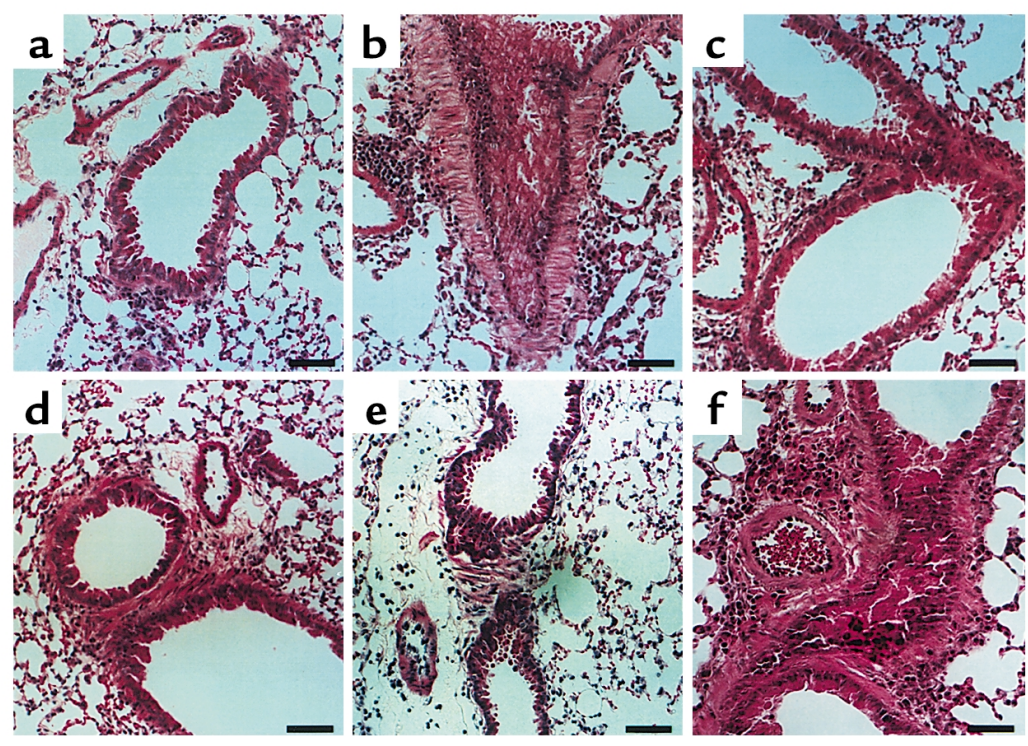


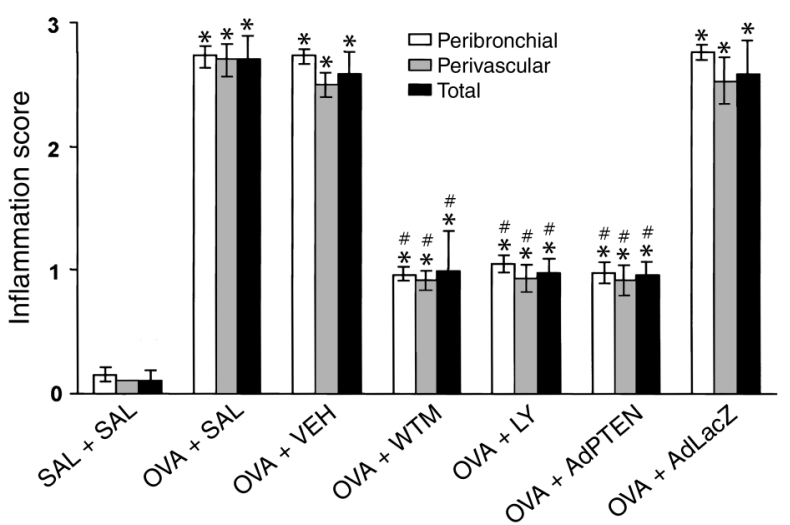

Figure 11

Effect of wortmannin, LY-294002, or AdPTEN on peribronchial and perivascular lung inflammation. Peribronchial, perivascular, and total lung inflammation were measured 72 hours after the last challenge in mice treated as described in Figure 4 legend. Bars represent mean \pm SD from six independent experiments. Total lung inflammation was defined as the average of the peribronchial and perivascular inflammation scores. ${ }^{*} P<0.05$ vs. SAL + SAL; ${ }^{\#} P<0.05$ vs. OVA + SAL and OVA + AdLacZ.

of $\beta$-gal-stained eosinophils at 24,48 , and 72 hours after the last airway challenge with OVA were $9.7 \%, 1.3 \%$, and $0 \%$ of total eosinophils, respectively (Figure 8).

Effect of wortmannin, LY-294002, or AdPTEN on cellular changes in BAL fluids. Numbers of total cells, eosinophils, lymphocytes, and neutrophils were increased significantly 72 hours after OVA inhalation compared with levels after saline inhalation (Figure 9). The increased numbers of eosinophils in BAL fluids at 3 days after OVA inhalation were significantly reduced by the intratracheal administration of wortmannin, LY-294002, or AdPTEN.

Effect of wortmannin, LY-294002, or AdPTEN on pathologic changes of OVA-induced asthma. Histologic analyses revealed typical pathologic features of asthma in the OVA-exposed mice. Numerous inflammatory cells, including eosinophils, infiltrated around the bronchioles, the airway epithelium was thickened, and mucus and debris had accumulated in the lumen of bronchioles (Figure 10b). Mice treated with wortmannin
(Figure 10c), LY-294002 (Figure 10d), or AdPTEN (Figure $10 \mathrm{e}$ ) showed marked reductions in the thickening of airway epithelium, in the infiltration of inflammatory cells in the peribronchiolar region, in the number of inflammatory cells, and in the amount of debris in the airway lumen. In contrast, no significant changes were observed in AdLacZ-treated mice (Figure 10f).

The scores of peribronchial, perivascular, and total lung inflammation were increased significantly 72 hours after OVA inhalation compared with scores after saline inhalation (Figure 11). The increased peribronchial, perivascular, and total lung inflammation after OVA inhalation was significantly reduced by intratracheal administration of wortmannin, LY-294002, or AdPTEN. These results suggest that wortmannin, LY-294002, and AdPTEN inhibit antigen-induced inflammation in the lungs, including the influx of eosinophils.

Effect of wortmannin, LY-294002, or AdPTEN on levels of IL-4, IL-5, and ECP in BAL fluids. Levels of IL-4 and IL-5 were increased significantly at 72 hours after OVA inhalation compared with levels after saline inhalation (Figure 12 , $a$ and $b$ ). The increased IL-4 and IL-5 levels in BAL fluids at 72 hours after OVA inhalation were significantly reduced by the intratracheal administration of wortmannin, LY-294002, or AdPTEN. In addition, the increased ECP levels in the BAL fluids at 72 hours after OVA inhalation were significantly reduced by the administration of wortmannin, LY-294002, or AdPTEN (Figure 12c). These results suggest that wortmannin, LY-294002, and AdPTEN inhibit the antigen-induced release of soluble mediators of inflammation into the lungs.

Effect of wortmannin, LY-294002, or AdPTEN on airway byperresponsiveness. Airway responsiveness was assessed as a percent increase of Penh in response to increasing doses of methacholine. In OVA-sensitized and -challenged mice, the dose-response curve of percent Penh shifted to the left compared with that of control mice (Figure 13). In addition, the percent Penh produced by methacholine administration (at doses from 2.5 to 50 $\mathrm{mg} / \mathrm{ml}$ ) increased significantly in the OVA-sensitized and -challenged mice compared with the controls.
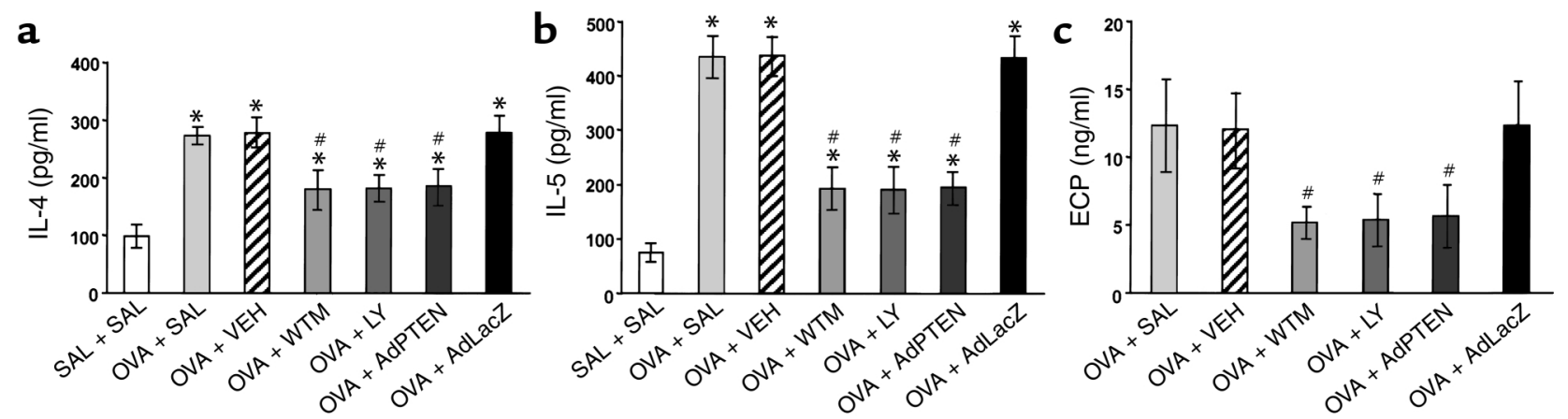

Figure 12

Effect of wortmannin, LY-294002, or AdPTEN on IL-4, IL-5, and ECP levels in BAL fluids of OVA-sensitized and -challenged mice. Enzyme immunoassay of IL-4 (a), IL-5 (b), and ECP (c). Sampling was performed 72 hours after the last challenge in mice treated as described in Figure 4 legend. Bars represent mean \pm SD from six independent experiments. ${ }^{*} P<0.05$ vs. SAL $+\mathrm{SAL} ;{ }^{\#} P<0.05$ vs. OVA + SAL and OVA + AdLacZ. 


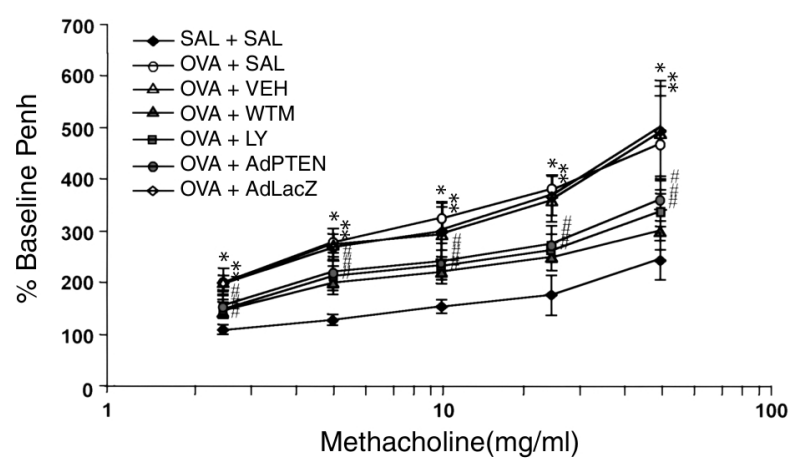

\section{Figure 13}

Effect of wortmannin, LY-294002, or AdPTEN on airway responsiveness in OVA-sensitized and -challenged mice. Airway responsiveness was measured 72 hours after the last challenge in mice treated as described in Figure 4 legend. Airway responsiveness to aerosolized methacholine was measured in unrestrained, conscious mice. Mice were placed in the main chamber of a barometric plethysmograph and nebulized first with saline and then with increasing doses (from 2.5 to $50 \mathrm{mg} / \mathrm{ml}$ ) of methacholine for 3 minutes for each nebulization. Readings of breathing parameters were taken for 3 minutes after each nebulization, during which time Penh values were determined. Data represent mean \pm SD from six independent experiments. ${ }^{*} P<0.05 \mathrm{vs}$. SAL $+\mathrm{SAL}$; ${ }^{\#} P<0.05$ vs. OVA + SAL and OVA + AdLacZ.

OVA-sensitized and -challenged mice treated with wortmannin, LY-294002, or AdPTEN showed a doseresponse curve of percent Penh that shifted to the right compared with that of untreated mice or OVAsensitized and -challenged mice treated with AdLacZ. These results indicate that wortmannin, LY-294002, or AdPTEN treatment reduces OVA-induced airway hyperresponsiveness.

\section{Discussion}

To our knowledge, this report is the first to describe the involvement of PTEN in airway hyperresponsiveness and inflammation in a murine model of asthma. We found that administration of PI3K inhibitors or AdPTEN reduced bronchial inflammation and airway hyperresponsiveness.

Bronchial asthma is an inflammatory disease of the airways characterized by airway obstruction and increased airway responsiveness. Eosinophils play important roles in the pathophysiology of asthma (2). Although considerable controversy remains regarding the relationship between bronchial eosinophilic inflammation and airway hyperresponsiveness, it is believed that the eosinophils degranulate to release toxic granule proteins (19) and that these products can cause airway hyperresponsiveness.

Many inflammatory mediators attract and activate eosinophils via signal transduction pathways involving the enzyme PI3K (3-7). In this study we found that PI3K activity increased significantly after allergen challenge in a murine model of asthma. Administration of either wortmannin or LY-294002, which are both potent and selective PI3K inhibitors, reduced eosinophilic inflammation and airway hyperresponsiveness. We also found that the increased ECP levels in BAL fluids measured 72 hours after OVA inhalation were significantly reduced by the intratracheal administration of wortmannin or LY-294002. In addition, levels of IL-4 and IL-5 were increased greatly at 72 hours after OVA inhalation compared with levels after saline inhalation. The increased IL-4 and IL-5 levels in BAL fluids at 72 hours after OVA inhalation were significantly reduced by the intratracheal administration of wortmannin or LY-294002.

Our data are consistent with the results obtained in a study reported by Tigani et al. (7) showing that wortmannin given at high concentrations $(100 \mu \mathrm{g} / \mathrm{kg})$ inhibits the increased number of eosinophils and eosinophil peroxidase activity in the BAL fluid of OVAchallenged animals. Palframan et al. (5) reported that wortmannin inhibited IL-5-induced selective release of eosinophils from perfused bone marrow, as well as selective eosinophil chemokinesis in vitro. The use of PI3K inhibitors has revealed the involvement of PI3K in the transduction of activation signals generated by many inflammatory mediators in eosinophils. Therefore, we conclude that PI3K may play an important role in the induction and maintenance of asthma.

PTEN is one of the most frequently mutated tumor suppressors in human cancer. It is also essential for embryonic development, cell migration, and apoptosis $(8,20,21)$. PTEN has been implicated in regulating cell survival signaling through the PI3K/Akt pathway $(8,9$, 22-24). PTEN dephosphorylates the $\mathrm{D} 3$ position of the key lipid second messenger PIP3 (8). In addition, PTEN has weak protein tyrosine phosphatase activity, which may target focal adhesion kinase (FAK) and/or Shc and thereby modulate other complex pathways. However, the major function of PTEN appears to be downregulation of the PI3K product PIP3. In this study, PTEN protein expression and PTEN activity were decreased in OVA-induced asthma. Immunoreactive PTEN localized in epithelial layers around the bronchioles of control mice; this PTEN rapidly disappeared in allergeninduced asthmatic lungs. The epithelial layer is one of the sites of the effect since this is the site of reduced PTEN expression and expression rescued with AdPTEN. These results suggest that once insulted, the epithelia may attract eosinophils and that this recruitment is impeded by inhibitors of PI3K.

We also found that immunoreactive $\beta$-gal localized in epithelial layers around the bronchioles from 24 to 72 hours after the last airway challenge with OVA. These $\beta$-gal-stained eosinophils were decreased in a time-dependent manner. Therefore, these data are consistent with AdPTEN affecting epithelial stimulation of eosinophil function. However, the data do not indicate a direct effect of AdPTEN on eosinophils. Immunocytologic analysis of BAL fluids showed localization of immunoreactive PTEN in BAL cells from control mice. However, immunoreactive PTEN was markedly reduced in precipitated cells, especially 
eosinophils, from OVA-exposed mice. Intratracheal administration of AdPTEN restored immunoreactive PTEN expression in BAL eosinophils. The increased ECP levels in BAL fluids at 72 hours after OVA inhalation were significantly reduced by the intratracheal administration of AdPTEN.

Several reports have shown that PI3K inhibitors are potent inhibitors of eosinophil degranulation (3-7). Wortmannin, a PI3K inhibitor, potently inhibits peroxidase release from eosinophils (6) and blocks neuropeptide-induced chemotaxis and IL-5-induced $\beta 2$-integrin-dependent adhesion to ICAM-1-coated surfaces in human eosinophils $(3,4)$.

Based on the effectiveness of PI3K inhibitors and the decreased expression of PTEN in asthmatic mice, we administered AdPTEN to examine its effect. AdPTEN was effective in reducing all signs of asthma examined. One likely mechanism for the effectiveness of AdPTEN is that administration of PTEN, which blocks the action of PI3K, might reduce eosinophilic inflammation and airway hyperresponsiveness in allergen-challenged mice, probably by inhibiting eosinophil degranulation and suppression of IL-4 and IL-5 concentrations in the airway.

Our results strongly indicate that alterations in PTEN levels are implicated in the pathogenesis of bronchial asthma.

\section{Acknowledgments}

This study was supported by a grant (02-PJ1-PG1CH01-0006) from the Korea Health 21 Research and Development Project, Ministry of Health and Welfare, Republic of Korea.

1. Kay, A.B. 1991. Asthma and inflammation. J. Allergy Clin. Immunol. 87:893-910.

2. Frigas, E., and Gleich, G.J. 1986. The eosinophil and the pathophysiology of asthma. J. Allergy Clin. Immunol. 77:527-537.

3. Dunzendorfer, S., Meierhofer, C., and Wiedermann, C.J. 1998. Signaling in neuropeptide-induced migration of human eosinophils. J. Leukoc. Biol. 64:828-834.

4. Zhu, X., et al. 2000. A surrogate method for assessment of beta (2)-integrin-dependent adhesion of eosinophils to ICAM-1. J. Immunol. Meth. 240:157-164.

5. Palframan, R.T., et al. 1998. Mechanisms of acute eosinophil mobilization from the bone marrow stimulated by interleukin 5: the role of specific adhesion molecules and phosphatidylinositol 3-kinase. J. Exp. Med. 188:1621-1632.

6. Ezeamuzie, C.I., Sukumaran, J., and Philips, E. 2001. Effect of wortmannin on human eosinophil responses in vitro and on bronchial inflammation and airway hyperresponsiveness in guinea pigs in vivo. Am. J. Respir. Crit. Care Med. 164:1633-1639.

7. Tigani, B., Hannon, J.P., Mazzoni, L., and Fozard, J.R. 2001. Effects of wortmannin on airways inflammation induced by allergen in actively sensitised Brown Norway rats. Eur. J. Pharmacol. 433:217-223.

8. Yamada, K.M., and Araki, M. 2001. Tumor suppressor PTEN: modulator of cell signaling, growth, migration and apoptosis. J. Cell Sci. 114:2375-2382.

9. Cantley, L.C., and Neel, B.G. 1999. New insights into tumor suppression: PTEN suppresses tumor formation by restraining the phosphoinositide 3-kinase/AKT pathway. Proc. Natl. Acad. Sci. U. S. A. 96:4240-4245.

10. Freund, G.G., Kulas, D.T., and Mooney, R.A. 1993. Insulin and IGF-1 increase mitogenesis and glucose metabolism in the multiple myeloma cell line, RPMI8226. J. Immunol. 151:1811-1820.

11. Minshall, C., Arkins, S., Freund, G.G., and Kelley, K.W. 1996. Requirement for phosphatidylinositol 3'-kinase to protect hemopoietic progenitors against apoptosis depends upon the extracellular survival factor. J. Immunol. 156:939-947.

12. He, T.C., et al. 1998. A simplified system for generating recombinant adenoviruses. Proc. Natl. Acad. Sci. U. S. A. 95:2509-2514.

13. Hwang, P.H., et al. 2001. Suppression of tumorigenicity and metastasis in B16F10 cells by PTEN/MMAC1/TEP1 gene. Cancer Lett. 172:83-91.

14. Alves, A.C., et al. 1996. Selective inhibition of phosphodiesterase type IV suppresses the chemotactic responsiveness of rat eosinophils in vitro. Eur. J. Pharmacol. 312:89-96.

15. Hamelmann, E., et al. 1997. Noninvasive measurement of airway responsiveness in allergic mice using barometric plethysmography. Am. J. Respir. Crit. Care Med. 156:766-775.

16. Lee, Y.C., Kwak, Y.G., and Song, C.H. 2002. Contribution of vascular endothelial growth factor to airway hyper-responsiveness and inflammation in a murine model of toluene diisocyanate-induced asthma. J. Immunol. 168:3595-3600.

17. Tournoy, K.G., Kips, J.C., Schou, C., and Pauwels, R.A. 2000. Airway eosinophilia is not a requirement for allergen-induced airway hyperresponsiveness. Clin. Exp. Allergy. 30:79-85.

18. Teramoto, S., et al. 1998. Investigation of effects of anesthesia and age on aspiration in mice through LacZ gene transfer by recombinant E1deleted adenovirus vectors. Am. J. Respir. Crit. Care Med. 158:1914-1919.

19. Gleich, G.J. 2000. Mechanisms of eosinophil-associated inflammation. J. Allergy Clin. Immunol. 105:652-663.

20. Lu, Y., et al. 1999. The PTEN/MMAC1/TEP tumor suppressor gene decreases cell growth and induces apoptosis and anoikis in breast cancer cells. Oncogene. 18:7034-7045.

21. Di Cristofano, A., Pesce, B., Cordon-Cardo, C., and Pandolfi, P.P. 1998. Pten is essential for embryonic development and tumour suppression. Nat. Genet. 19:348-355.

22. Maehama, T., and Dixon, J.E. 1998. The tumor suppressor, PTEN/MMAC1, dephosphorylates the lipid second messenger, phosphatidylinositol 3,4,5-trisphosphate. J. Biol. Chem. 273:13375-13378.

23. Sun, H., et al. 1999. PTEN modulates cell cycle progression and cell survival by regulating phosphatidylinositol 3,4,5-trisphosphate and Akt/protein kinase B signaling pathway. Proc. Natl. Acad. Sci. U. S. A. 96:6199-6204.

24. Myers, M.P., et al. 1998. The lipid phosphatase activity of PTEN is critical for its tumor suppressor function. Proc. Natl. Acad. Sci. U. S. A. 95:13513-13518. 